\title{
Predicting subretinal fluid absorption with machine learning in patients with central serous chorioretinopathy
}

\author{
Fabao Xu ${ }^{1 \#}$, Yifan Xiang ${ }^{1 \#}$, Cheng Wan ${ }^{2 \#}$, Qijing You ${ }^{2}$, Lijun Zhou ${ }^{1}$, Cong Li $^{1}$, Songjian Gong ${ }^{3}$, \\ Yajun Gong ${ }^{1}$, Longhui $\mathrm{Li}^{1}$, Zhongwen $\mathrm{Li}^{1}$, Li Zhang ${ }^{4}$, Xiayin Zhang ${ }^{1}$, Chong Guo ${ }^{1}$, Kunbei Lai ${ }^{1}$, \\ Chuangxin Huang ${ }^{1}$, Hongkun Zhao ${ }^{1}$, Chenjin Jin ${ }^{1}$, Haotian Lin $^{1,5}$
}

${ }^{1}$ State Key Laboratory of Ophthalmology, Zhongshan Ophthalmic Center, Sun Yat-Sen University, Guangzhou, China; ${ }^{2}$ Nanjing University of Aeronautics and Astronautics, Nanjing, China; ${ }^{3}$ Xiamen Eye Center, Affiliated to Xiamen University, Xiamen, China; ${ }^{4}$ Department of Ophthalmology, The Central Hospital of Wuhan, Tongji Medical College, Huazhong University of Science and Technology, Wuhan, China; ${ }^{5}$ Center of Precision Medicine, Sun Yat-Sen University, Guangzhou, China

Contributions: (I) Conception and design: H Lin, C Jin, F Xu; (II) Administrative support: H Lin, C Jin; (III) Provision of study materials or patients: H Lin, C Jin, F Xu, Y Xiang; (IV) Collection and assembly of data: C Jin, F Xu, C Wan, L Zhou, C Li, S Gong, Y Gong, C Huang, H Zhao; (V) Data analysis and interpretation: Q You, F Xu, L Li, Z Li, L Zhang, X Zhang; (VI) Manuscript writing: All authors; (VII) Final approval of manuscript: All authors.

"These authors contributed equally to this work.

Correspondence to: Chenjin Jin. State Key Laboratory of Ophthalmology, Zhongshan Ophthalmic Center, Sun Yat-Sen University, Guangzhou, China. Email: jinchj@mail.sysu.edu.cn; Haotian Lin. State Key Laboratory of Ophthalmology, Zhongshan Ophthalmic Center, Sun Yat-Sen University, Guangzhou, China. Email: haot.lin@hotmail.com.

Background: Machine learning was used to predict subretinal fluid absorption (SFA) at 1, 3 and 6 months after laser treatment in patients with central serous chorioretinopathy (CSC).

Methods: The clinical and imaging data from 480 eyes of 461 patients with CSC were collected at Zhongshan Ophthalmic Center (ZOC) and Xiamen Eye Center (XEC). The data included clinical features from electronic medical records and measured features from fundus fluorescein angiography (FFA), indocyanine green angiography (ICGA), optical coherence tomography angiography (OCTA), and optical coherence tomography (OCT). A ZOC dataset was used for training and internal validation. An XEC dataset was used for external validation. Six machine learning algorithms and a blending algorithm were trained to predict SFA in patients with CSC after laser treatment. The SFA results predicted by machine learning were compared with the actual patient prognoses. Based on the initial detailed investigation, we constructed a simplified model using fewer clinical features and OCT features for convenient application.

Results: During the internal validation, random forest performed best in SFA prediction, with accuracies of $0.651 \pm 0.068,0.753 \pm 0.065$ and $0.818 \pm 0.058$ at 1,3 and 6 months, respectively. In the external validation, XGBoost performed best at SFA prediction with accuracies of $0.734,0.727$, and 0.900 at 1,3 and 6 months, respectively. The simplified model showed a comparable level of predictive power.

Conclusions: Machine learning can achieve high accuracy in long-term SFA predictions and identify the features relevant to CSC patients' prognoses. Our study provides an individualized reference for ophthalmologists to treat and create a follow-up schedule for CSC patients.

Keywords: Machine learning; central serous chorioretinopathy (CSC); laser treatment; subretinal fluid absorption (SFA); optical coherence tomography (OCT)

Submitted Feb 11, 2020. Accepted for publication Nov 08, 2020.

doi: 10.21037/atm-20-1519

View this article at: http://dx.doi.org/10.21037/atm-20-1519 


\section{Introduction}

Central serous chorioretinopathy (CSC) is a retinochoroid disease that causes idiopathic serous retinal detachment, which is associated with one or more leakages from the choroid through the defects in the retinal pigment epithelium (RPE) outer blood-retina barrier. It primarily affects relatively young men of working age $(1,2)$. CSC is fairly common, being considered as the fourth most prevalent non-surgical retinopathy associated with subretinal fluid (SRF) leakage in the world (3). Although SRF can resolve spontaneously in some cases, many patients still suffer permanent vision loss or significant clinical sequelae due to incomplete subretinal fluid absorption (SFA) (2).

CSC is usually divided into two categories: acute CSC and chronic CSC, based on the duration of symptoms. Most investigators have employed this incomplete and relatively rudimentary classification of CSC in their clinical studies (2). However, there is no clear consensus regarding the criteria for classification. Our lack of an established classification system necessitates studying the natural disease progression of CSC and its therapeutic management $(4,5)$. There remains an absence of academically recognized treatment guidelines for CSC. Ophthalmologists have to make decisions experientially in the treatment of CSC patients.

To develop more precise care for patients, we have established an individualized management plan based on SFA utilizing big data. SFA is the most concerning issue for clinicians after treatment, and it is the most important prognostic characteristic for patients with CSC (6). The increase or decrease in SFA affects the therapeutic strategy and the follow-up intervals for patients. In our study, we tried to establish an intelligent prediction system to foresee SFA at 1, 3 and 6 months after laser treatment with big data that incorporates medical records and imaging features, which help us to clarify the prognosis of patients with CSC and choose a sensible treatment.

We present the following article in accordance with the TRIPOD reporting checklist (available at http://dx.doi. org/10.21037/atm-20-1519).

\section{Methods}

\section{Data collection}

CSC was defined as patients with leakage on their fundus fluorescein angiography (FFA), abnormal choroidal circulation such as hyperpermeability, dilated choroidal vessels or other abnormal microangiopathy on indocyanine green angiography (ICGA), and serous retinal detachment (SRD) as confirmed by optical coherence tomography angiography (OCTA) and optical coherence tomography (OCT). A total of 416 eyes in 401 patients and 64 eyes in 60 patients were studied at Zhongshan Ophthalmic Center (ZOC) and Xiamen Eye Center (XEC), respectively, from January 2013 to September 2019. A definite diagnosis was made for all patients based on FFA and ICGA. The patients were followed for 1-6 months after treatment. Study exclusion criteria were as follows: (I) patients with high myopia, as defined as a refractive error (spherical equivalent) $<-6.00$ diopters, or an axial length $>26.5 \mathrm{~mm}$ and (II) patients with media opacities or signal strength indexes who were affected. The study was conducted in accordance with the Declaration of Helsinki (as revised in 2013). The study was approved by the ethics board of ZOC (No. 2020KYPJ024) and individual consent for this retrospective analysis was waived.

A total of 6,732 imaging pictures (1,248 FFA, 1,248 ICGA, 1,412 OCTA, and 2,824 OCT images) and 554 imaging pictures (192 FFA and 362 OCT images) were collected from ZOC and XEC, respectively. FFA (Heidelberg Spectralis, Heidelberg, Germany) and ICGA (Heidelberg Spectralis, Heidelberg, Germany) images for each patient were included only at the baseline, including three images from the early, middle and late phases. However, for the OCTA (RTVue XR Avanti with AngioVue; Optovue Inc., Fremont, CA, USA) and OCT (Heidelberg Spectralis, Heidelberg, Germany) follow-up data, data from the baseline, 1, 3, and 6 months after laser treatment were included. Measurement information on the FFA, ICGA, OCTA, and OCT, were extracted from the software of Heidelberg Eye Explorer (version 1.7.1.0) and Optovue (Version 2017.1.0.155). The clinical features (20 clinical features, e.g., the duration of CSC) of these CSC patients were also extracted from electronic medical records (details are provided in Table S1). For therapy information, the data from ZOC included conventional laser (CL) therapy (117 eyes), subthreshold micropulse laser (SML) therapy (80 eyes) and half-dose photodynamic therapy (hd-PDT) (219 eyes). The XEC data included CL therapy (21 eyes), $577 \mathrm{~nm}$ SML therapy (14 eyes) and hd-PDT (29 eyes). According to type 3 of the TRIPOD statement, we developed prediction models using the dataset from $\mathrm{ZOC}$ and evaluated its performance in a separate dataset from XEC (7).

We treated each eye as a separate CSC case during data preprocessing. There were only a few values missing 

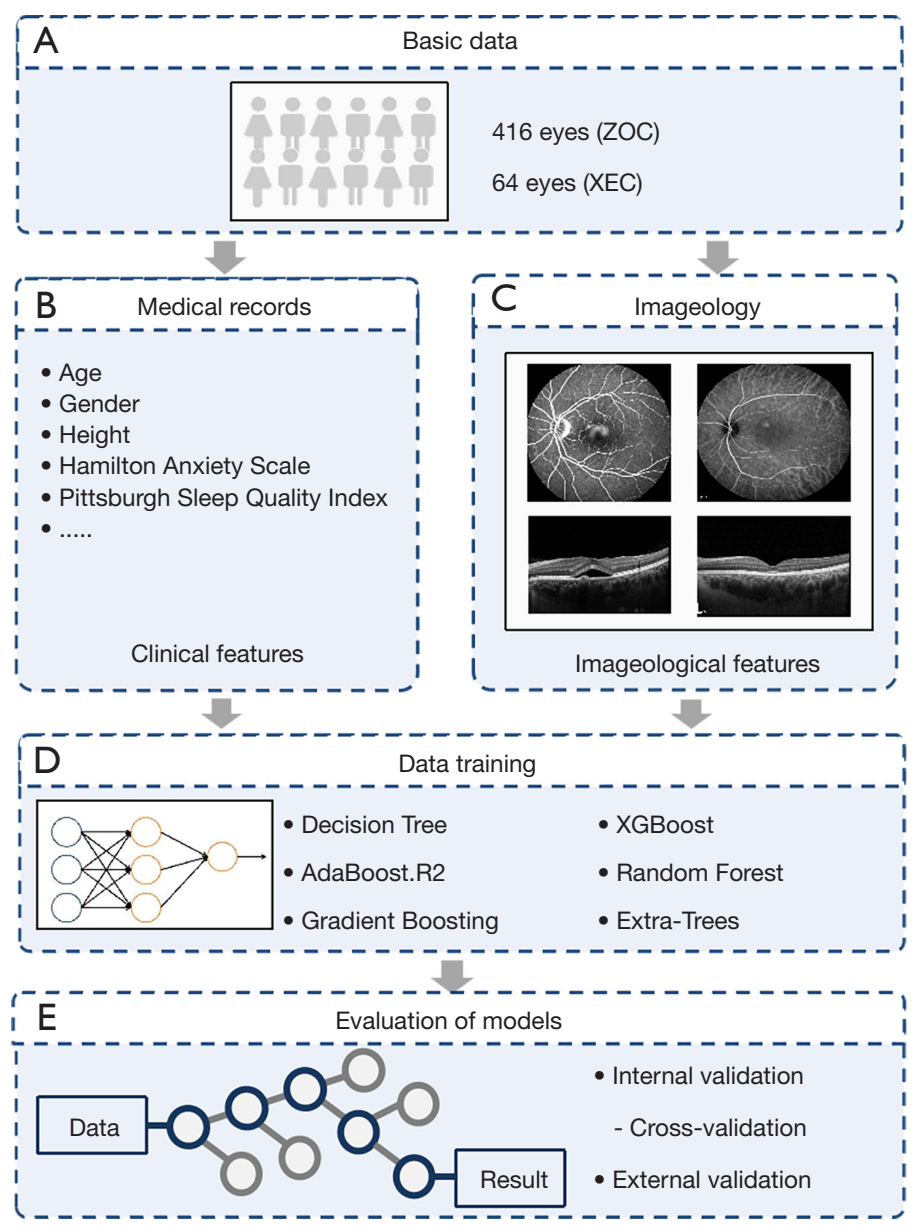

Figure 1 Overall Study Workflow. Workflow diagram showing the training overview for the SFA prediction model.

from the ZOC data, and we filled them in with the mean values for other cases. However, all the ICGA and OCTA features were missing for patients collected from XEC data. Considering their clinical significance and importance in the algorithm, we ultimately chose to fill in the missing features in the XEC data with the mean values for the same features of the ZOC data.

\section{Construction of models}

All the training and testing approaches were run on a workstation configured with 32-core Intel Xeon E5 CPU and 128 GB RAM. We used Python 3.6.8 in the Ubuntu 16.04 system. The Python libraries we used in this study are as follows: Jupyter (1.1.0), Scikit-Learn (0.19.1), and Pandas (0.20.3). Six separate algorithms were trained, with a total of 165 features and state-of-the-art performance in each adaptive domain, and they are listed as follows:
Decision Tree (8), AdaBoost.R2 (9), Gradient Boosting (10), XGBoost (11), random forest (12), and extra-trees (13). While selecting the optimal algorithms, we randomly divided the ZOC data into 10 parts, and calculated the decision tree, AdaBoost.R2, gradient boosting, XGBoost, random forest, and extra-trees by 10 -fold cross-validation. We then selected the best three algorithms for the ensemble according to the prediction accuracy. After determining the selected algorithms, to make the most of the existing clinical data, we used all the data from ZOC to retrain the selected algorithms. The workflow is shown in Figure 1.

For each of the above algorithms, we used a grid search with cross-validation to select the suitable hyperparameters (14).

The ensemble learning method was applied to obtain a model with good fitting ability and generalization performance for tasks such as classification and regression and model averaging is a common and effective approach $(14,15)$. 
Table 1 Patient demographics

\begin{tabular}{|c|c|c|c|c|c|c|}
\hline Variable & \multicolumn{2}{|c|}{$1 \mathrm{M}$ prediction } & \multicolumn{2}{|c|}{$3 \mathrm{M}$ prediction } & \multicolumn{2}{|c|}{$6 \mathrm{M}$ prediction } \\
\hline Patients & 401 (63 females) & 60 (11 females) & 308 (46 females) & 30 (5 females) & 244 (37 females) & 19 (2 females) \\
\hline Eyes & 416 & 64 & 322 & 33 & 258 & 20 \\
\hline Age (years) & $43.19 \pm 6.44$ & $43.86 \pm 7.06$ & $42.87 \pm 6.44$ & $43.21 \pm 7.51$ & $42.96 \pm 6.48$ & $41.70 \pm 6.73$ \\
\hline
\end{tabular}

Visual acuity (VA) values are presented as the means \pm standard deviations at baseline in different groups (in logarithm of minimum angle of resolution [logMAR] units). ZOC, Zhongshan Ophthalmic Center; XEC, Xiamen Eye Center.

\section{Evaluation of models}

To evaluate the performance of our models, the accuracy in predicting SFA at 1, 3 and 6 months after laser treatment were validated. The baseline data were used to predict SFA at 1, 3 and 6 months after treatment. To obtain more accurate predictions, when predicting SFA at 3 months after treatment, we trained the model using the baseline and 1-month data; to predict SFA at 6 months after treatment, we trained the models using the baseline, 1-month and 3 -month data. A 10-fold cross-validation was applied to evaluate the performance of the models.

\section{Simplified model}

We constructed a simplified prediction model using relatively few clinical data and OCT features to make our study more accessible for clinical use. The remaining features were determined according to the relative importance obtained during the establishment of the original algorithms (Figures S1-S6), and the difficulty in imaging feature acquisition. Table S2 shows all the training features of the simplified model. For the simplified model, the training steps are the same as those in the original models.

\section{Statistical analysis}

Accuracy (ACC) is to evaluate the predictive effectiveness of the model. Values are shown as means \pm SDs.

\section{Results}

A total of 480 eyes in 461 patients aged 28 to 71 years old (43.56 \pm 6.64$)$ were addressed during our study. The demographic information for the training and validation datasets are shown in Table 1. Table 2 shows the accuracies of predicting SFA during all the algorithm tasks. Among the original models, random forest had the best performance in the internal validation, and XGBoost performed best at external validation. For the simplified models, gradient boosting had the best performance for internal validation, and the blending algorithm performed best at external validation.

During the internal validation, random forest performed best in predicting SFA, with accuracies of $0.651 \pm 0.068$, $0.753 \pm 0.065$ and $0.818 \pm 0.058$ at 1,3 and 6 months, respectively. In the external validation, XGBoost performed best in predicting SFA, with accuracies of $0.734,0.727$, and 0.900 at 1,3 and 6 months, respectively. The simplified model showed a comparable level of predictive power. In the internal validation, gradient boosting performed best at predicting SFA, with accuracies of $0.630 \pm 0.057,0.780 \pm 0.043$ and $0.818 \pm 0.074$ at 1,3 and 6 months, respectively. During the external validation, the blending model performed best at predicting SFA, with accuracies of $0.656,0.758$, and 0.900 at 1,3 and 6 months, respectively.

In the cross-validation, random forest achieved highaccuracy predictions with areas under the curve (AUCs) ranging from 0.35 to 0.80 for 1 month, from 0.63 to 0.89 for 3 months, and from 0.92 to 1.00 for 6 months. In the external validation, XGBoost provided high-accuracy predictions with AUCs ranging from 0.76 to 1.00 for 1 month, from 0.27 to 0.72 for 3 months, and from 0.98 to 1.00 for 6 months (Figure 2). The simplified model exhibited an analogous prediction accuracy with AUCs ranging from 0.57 to 0.81 for 1 month, from 0.23 to 0.97 for 3 months, and from 0.88 to 1.00 for 6 months in the cross-validation, and AUCs ranging from 0.72 to 1.00 for 1 month, from 0.27 to 0.66 for 3 months, and from 0.98 to 1.00 for 6 months in the external validation (Figure 3). The distributions of prediction results and ground truth in each task are revealed 
Table 2 Accuracy of the subretinal fluid absorption predictions during internal and external validation tests

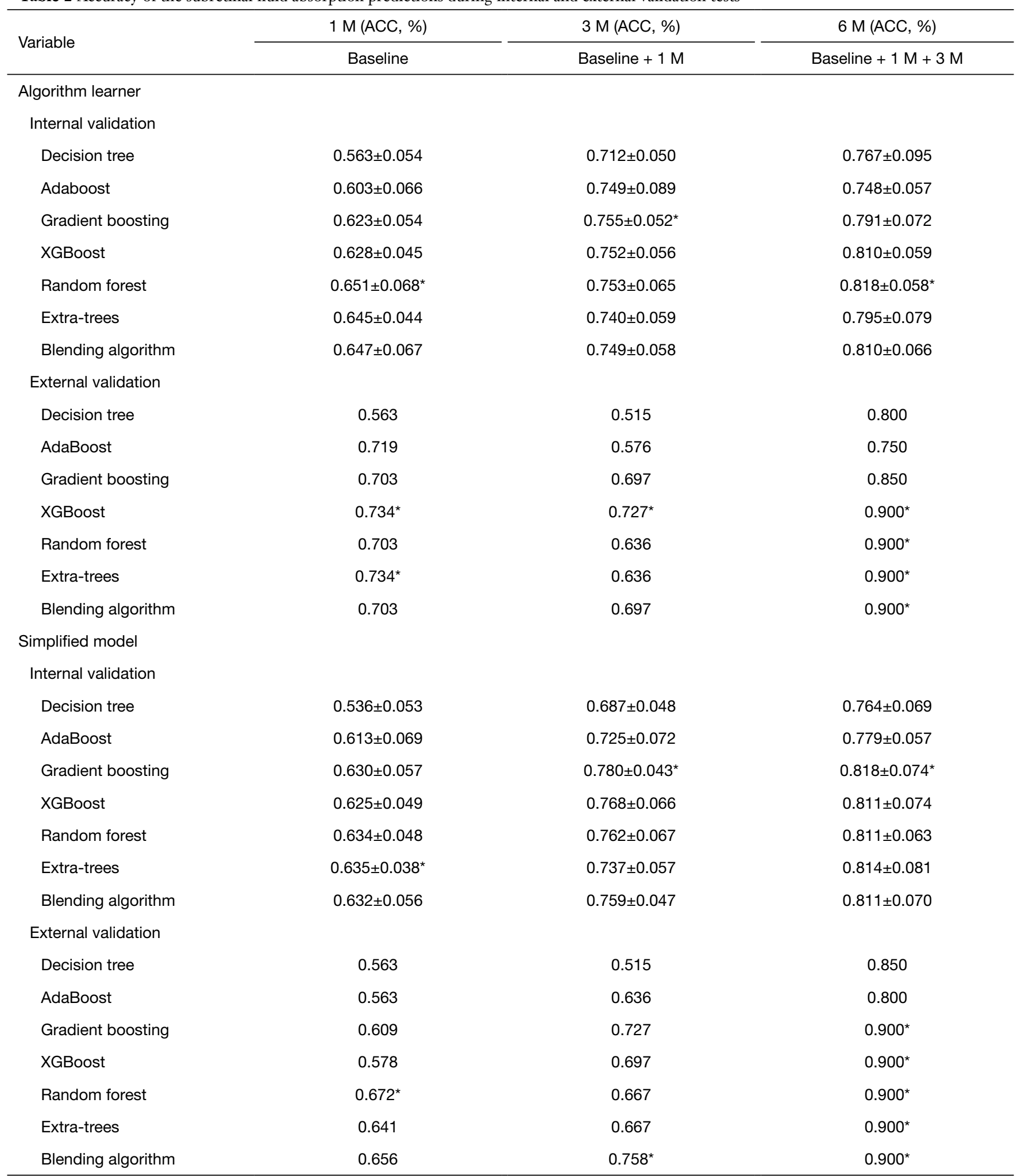

*, the best learners in all cases. ACC, accuracy of the SFA prediction at 1, 3 and 6 months after laser treatment compared with the ground truth. The results were stratified according to the follow-up periods and the points input into the algorithms. 

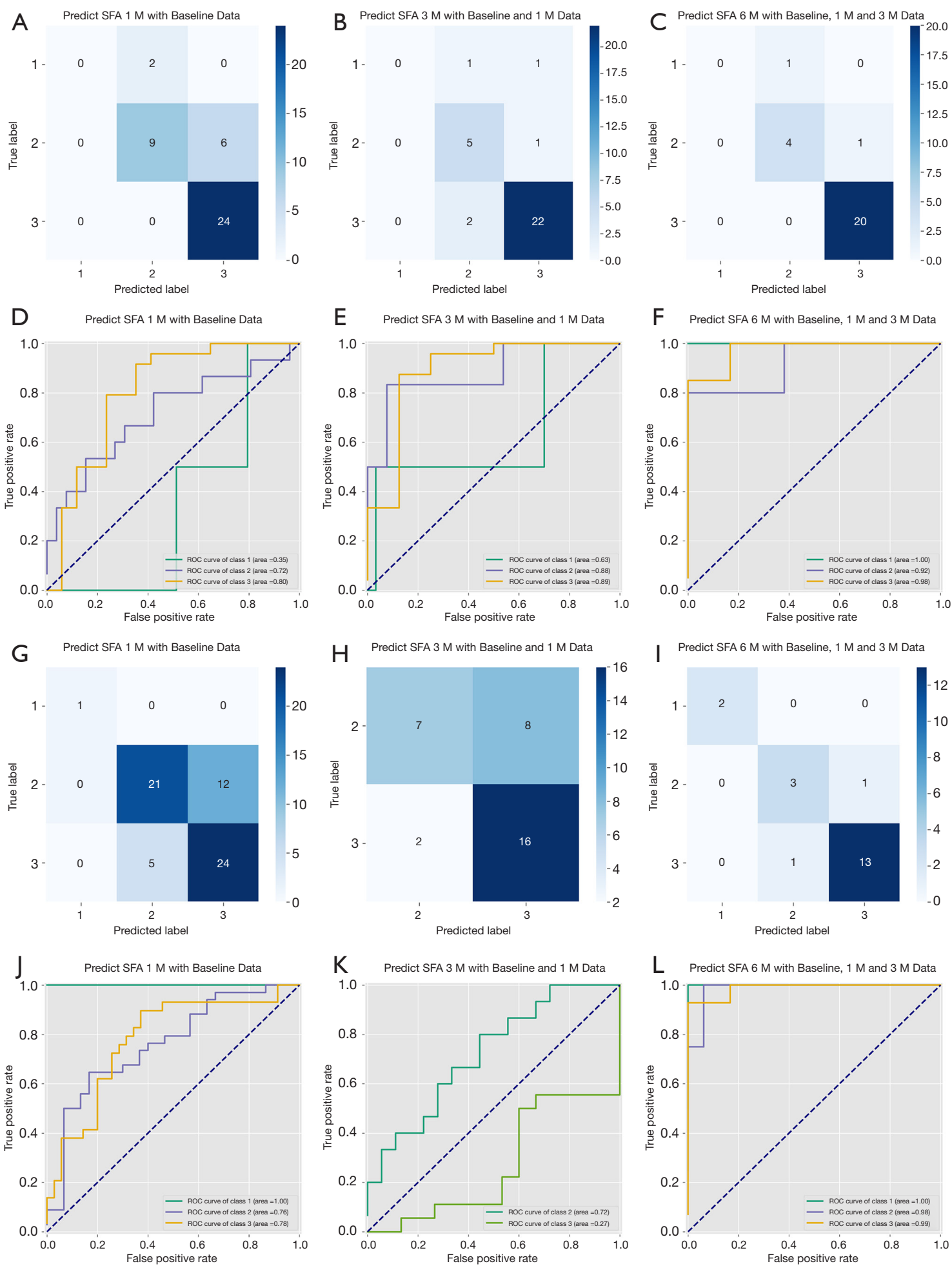

Figure 2 Prediction performance in the internal and external validation tests on the full model. Panels A, B, and C, CM of the classification in the internal validation test. Panels D, E, and F, ROC of the internal validation test. Panels G, H, and I, CM of the classification in the external validation test. Panels J, K, and L, ROC of the external validation test. CM, confusion matrix; ROC, receiver operating characteristic curve. 


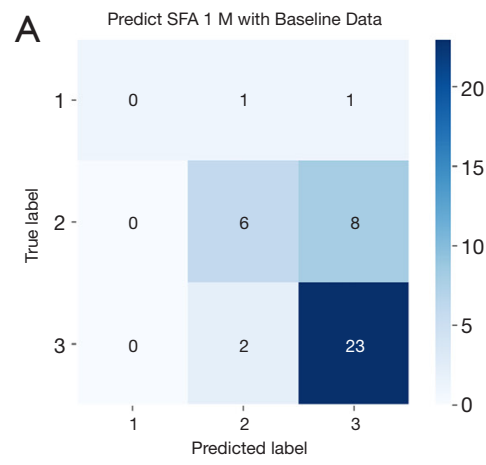

D

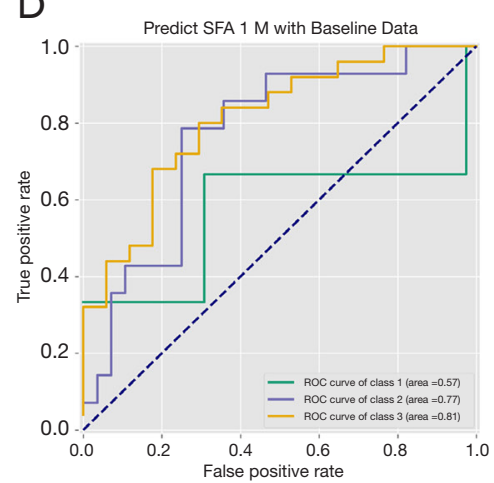

G

Predict SFA $1 \mathrm{M}$ with Baseline Data
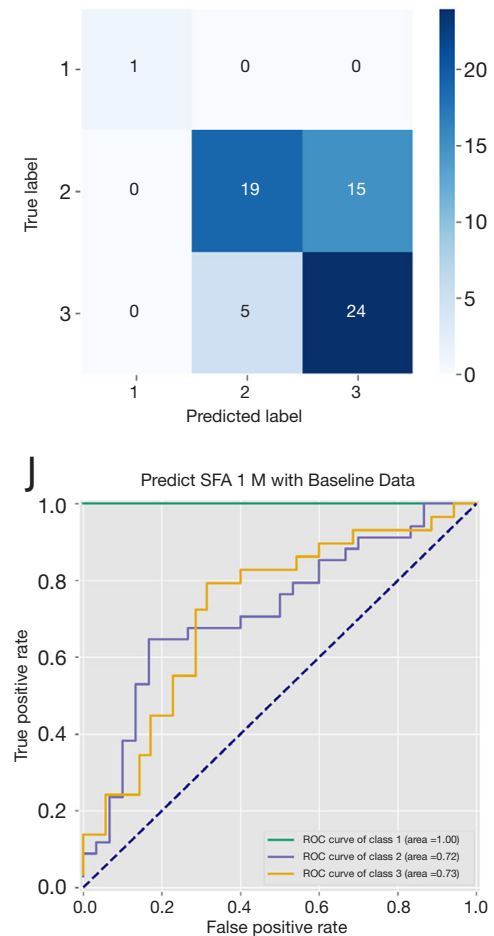

B Predict SFA $3 \mathrm{M}$ with Baseline and $1 \mathrm{M}$ Data

B

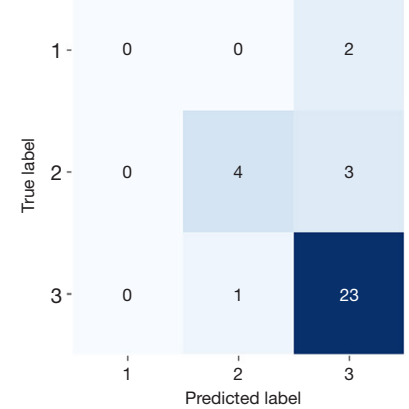

E

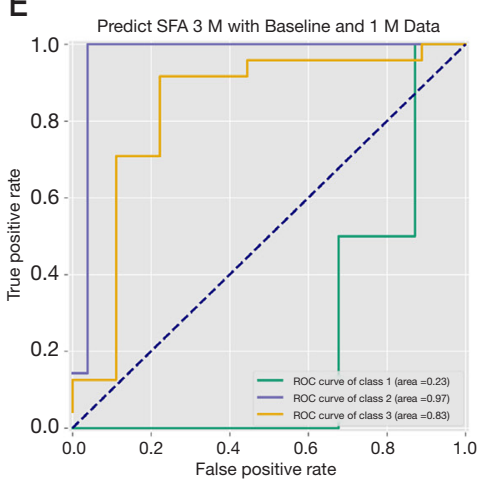

$\mathrm{H}$

H Predict SFA $3 \mathrm{M}$ with Baseline and $1 \mathrm{M}$ Data

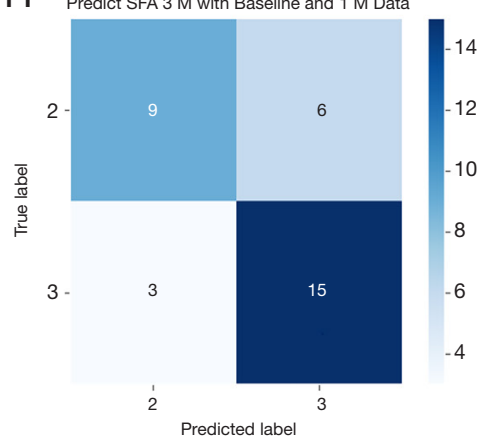

$\mathrm{K}$

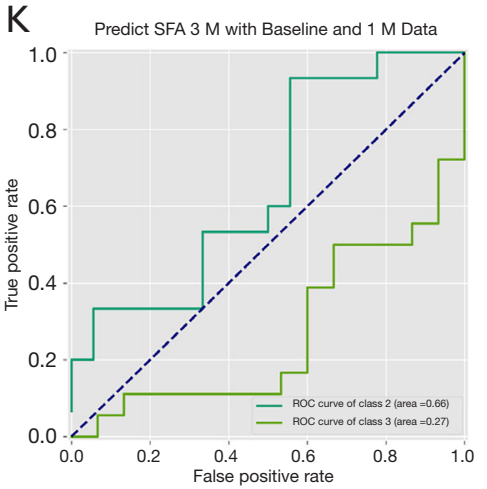

Predict SFA $6 \mathrm{M}$ with Baseline, $1 \mathrm{M}$ and $3 \mathrm{M}$ Data

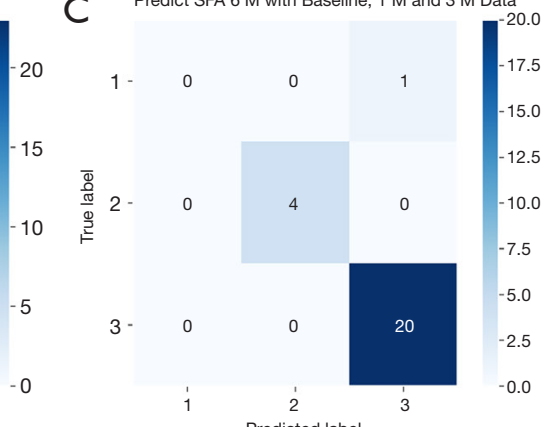

$\mathrm{F}$

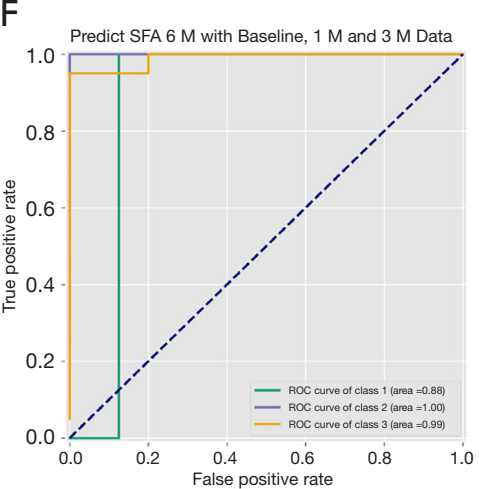

I Predict SFA 6 $\mathrm{M}$ with Baseline, $1 \mathrm{M}$ and $3 \mathrm{M}$ Data

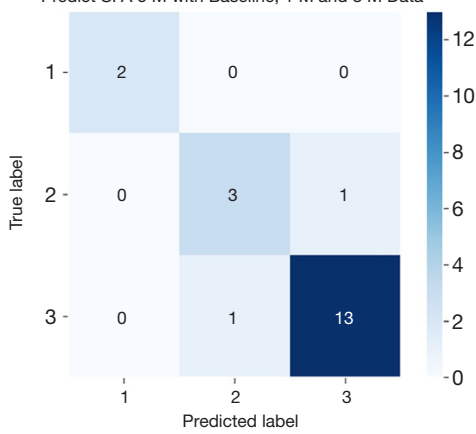

$\mathrm{L} \quad$ Predict SFA $6 \mathrm{M}$ with Baseline, $1 \mathrm{M}$ and $3 \mathrm{M}$ Data

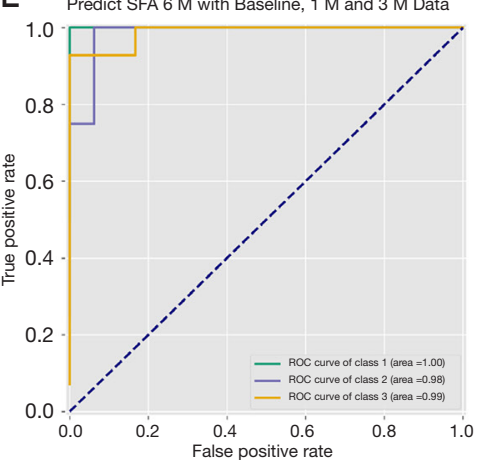

Figure 3 Prediction performance in the internal and external validation tests on the simplified model. Panels A, B, and C, CM of the classification in the internal validation test. Panels D, E, and F, ROC of the internal validation test. Panels G, H, and I, CM of the classification in the external validation test. Panels J, K, and L, ROC of the external validation test. CM, confusion matrix; ROC, receiver operating characteristic curve. 
in the confusion matrixes (CM) of Figure 2 and Figure 3. As shown in the receiver operating characteristic curve (ROC curve) and the CM, the SFA prediction at 6 months after treatment was the most accurate. Figures S1-S6 show the importance of features in the SFA predictions at 1,3 and 6 months.

\section{Discussion}

To our knowledge, no study has previously generated a machine learning model for predicting SFA in CSC patients. Our prediction models can foresee the patient's condition six months in advance. However, recent predictions within three months are relatively imprecise. Generally, short-term targets should be more accurate than long-term targets for applying artificial intelligence in disease prognosis prediction (16). This may be due to the lower limitation of the courses in the inclusion criteria; as the baseline data for CSC patients vary greatly, and the short-term consistency of patient prognosis is low. Usually, at six months after treatment, the SFA rates of CSC patients were much higher than at one and three months, which makes prediction tasks easier $(17,18)$. Last but not least, for prognosis predictions of complex fundus disease, we still need to accumulate follow-up data to improve our models.

By predicting SFA in patients, we can better understand the progression of CSC, choose cost-effective therapies and manage the follow-up more efficiently. The increasing use of FFA, ICGA, OCTA, and OCT in studying CSC has greatly improved our understanding of its pathogenesis and imaging characteristics. However, there is still no clear consensus regarding the criteria for classification and the guidelines for treatment. Our predictive system provides a reference for clinicians to choose therapies. We can input the information related to the patients and different therapies, and then the models will give us the probability of presenting SFA in six months. This is a new strategy for mining big data, and it enables us to achieve precise treatment without considering the specific classification of the disease. Based on the prediction models established in this study, we could choose a more efficient method such as hd-PDT in patients with consistent SRF, and we can also choose a more economical therapy such as SML for patients with SRF that is easily absorbed.

More than helping clinicians choose reasonable therapies, the models also help define the factors that are relevant to the CSC prognosis. In the analysis of feature importance, we found that in addition to the therapies chosen by clinicians, the baseline characteristics on the retina and lifestyle also exert a significant impact on SFA, including the SRF height, the central macula thickness (CMT), the double-layer sign (DLS), and the scores on the Pittsburgh Sleep Quality Index and Hamilton Anxiety Scale $(19,20)$. The findings can help us analyze the relevant factors that lead to different prognoses in CSC patients with the same treatment and guide patients to pay attention to influencing these factors in their daily life.

To make our study applicable to different scenarios, we constructed a simplified prediction model according to the relative importance obtained in the original models and the accessibility of the imaging features; the model is trained with only 11 clinical features and OCT features. This advantage can be further appreciated in applications by hospitals in underdeveloped areas without FFA, ICGA, and OCTA. In a clinical setting, FFA, ICGA, and OCTA are not necessary at certain stages, in most cases.

\section{Limitations}

There are some limitations in our present study. To improve the accuracy of the short-term predictions within three months, we need to incorporate the duration of the disease into the inclusion criteria. More data on CSC patients are necessary to improve the accuracy of the SFA prediction models. In addition, data for external validation is unicentric, and more real-world tests are needed for improved accuracy.

\section{Conclusions}

In summary, our study showed that multidimensional patterns of clinical and imaging features are predictive factors of SFA in CSC patients. The prediction models provide us with a whole new strategy to counsel patients from an individual-based perspective, and they serve as references for ophthalmologists who can choose efficient therapies and make follow-up schedules.

\section{Acknowledgments}

Funding: We received funding from the National Key R\&D Program of China (2018YFC0116500), the National Natural Science Foundation of China (81670866), the Major Project of Guangzhou Science and Technology Committee (201707020008), the National Natural Science Foundation of China (81770967), the National 
Natural Science Foundation of China (81822010), and the Jiangsu Planned Projects for Postdoctoral Research Funds (2019K226). The sponsors and funding organizations

\section{Footnote}

Reporting Checklist: The authors have completed the TRIPOD reporting checklist. Available at http://dx.doi. org/10.21037/atm-20-1519

Data Sharing Statement: Available at http://dx.doi. org/10.21037/atm-20-1519

Peer Review File: Available at http://dx.doi.org/10.21037/ atm-20-1519

Conflicts of Interest: All authors have completed the ICMJE uniform disclosure form (available at http://dx.doi. org/10.21037/atm-20-1519). The authors have no conflicts of interest to declare.

Ethical Statement: The authors are accountable for all aspects of the study in ensuring that questions related to the accuracy or integrity of any part of the work are appropriately investigated and resolved. The study was conducted in accordance with the Declaration of Helsinki (as revised in 2013). The study was approved by the ethics board of ZOC (No. 2020KYPJ024) and individual consent for this retrospective analysis was waived.

Open Access Statement: This is an Open Access article distributed in accordance with the Creative Commons Attribution-NonCommercial-NoDerivs 4.0 International License (CC BY-NC-ND 4.0), which permits the noncommercial replication and distribution of the article with the strict proviso that no changes or edits are made and the original work is properly cited (including links to both the formal publication through the relevant DOI and the license). See: https://creativecommons.org/licenses/by-nc-nd/4.0/.

\section{References}

1. Wong KH, Lau KP, Chhablani J, et al. Central serous chorioretinopathy: what we have learnt so far. Acta Ophthalmol 2016;94:321-5.

2. van Rijssen TJ, van Dijk EHC, Yzer S, et al. Central serous chorioretinopathy: Towards an evidence-based treatment guideline. Prog Retin Eye Res 2019;73:100770.
3. Wang M, Munch IC, Hasler PW, et al. Central serous chorioretinopathy. Acta Ophthalmol 2008;86:126-45.

4. Mohabati D, van Rijssen TJ, van Dijk EH, et al. Clinical characteristics and long-term visual outcome of severe phenotypes of chronic central serous chorioretinopathy. Clin Ophthalmol 2018;12:1061-70.

5. Otsuka S, Ohba N, Nakao K. A long-term follow-up study of severe variant of central serous chorioretinopathy. Retina 2002;22:25-32.

6. Manayath GJ, Ranjan R, Karandikar SS, et al. Central serous chorioretinopathy: Current update on management. Oman J Ophthalmol 2018;11:200-6.

7. Collins GS, Reitsma JB, Altman DG, et al. Transparent reporting of a multivariable prediction model for individual prognosis or diagnosis (TRIPOD): the TRIPOD statement. BMJ 2015;350:g7594.

8. Schoning V, Hammann F. How far have decision tree models come for data mining in drug discovery? Expert Opin Drug Discov 2018;13:1067-9.

9. Qi Z, Meng F, Tian Y, et al. Adaboost-LLP: A Boosting Method for Learning With Label Proportions. IEEE Trans Neural Netw Learn Syst 2018;29:3548-59.

10. Luo Y, Ye W, Zhao X, et al. Classification of Data from Electronic Nose Using Gradient Tree Boosting Algorithm. Sensors (Basel) 2017;17:2376.

11. Ogunleye AA, Qing-Guo W. XGBoost Model for Chronic Kidney Disease Diagnosis. IEEE/ACM Trans Comput Biol Bioinform 2019. [Epub ahead of print].

12. Pavey TG, Gilson ND, Gomersall SR, et al. Field evaluation of a random forest activity classifier for wristworn accelerometer data. J Sci Med Sport 2017;20:75-80.

13. Nattee C, Khamsemanan N, Lawtrakul L, et al. A novel prediction approach for antimalarial activities of Trimethoprim, Pyrimethamine, and Cycloguanil analogues using extremely randomized trees. J Mol Graph Model 2017;71:13-27.

14. Baskin, II. Machine Learning Methods in Computational Toxicology. Methods Mol Biol 2018;1800:119-39.

15. Zhang Y, Li M, Han S, et al. Intelligent Identification for Rock-Mineral Microscopic Images Using Ensemble Machine Learning Algorithms. Sensors (Basel) 2019;19:3914.

16. Rohm M, Tresp V, Muller M, et al. Predicting Visual Acuity by Using Machine Learning in Patients Treated for Neovascular Age-Related Macular Degeneration. Ophthalmology 2018;125:1028-36.

17. Zhou L, Chong V, Lai K, et al. A pilot prospective study of 577-nm yellow subthreshold micropulse laser treatment 
with two different power settings for acute central serous chorioretinopathy. Lasers Med Sci 2019;34:1345-51.

18. van Dijk EHC, Fauser S, Breukink MB, et al. Half-Dose Photodynamic Therapy versus High-Density Subthreshold Micropulse Laser Treatment in Patients with Chronic Central Serous Chorioretinopathy: The PLACE Trial. Ophthalmology 2018;125:1547-55.

Cite this article as: $\mathrm{Xu} F$, Xiang $\mathrm{Y}$, Wan C, You Q, Zhou L, Li C, Gong S, Gong Y, Li L, Li Z, Zhang L, Zhang X, Guo C, Lai K, Huang C, Zhao H, Jin C, Lin H. Predicting subretinal fluid absorption with machine learning in patients with central serous chorioretinopathy. Ann Transl Med 2021;9(3):242. doi: 10.21037/atm-20-1519
19. Manzar MD, BaHammam AS, Hameed UA, et al. Dimensionality of the Pittsburgh Sleep Quality Index: a systematic review. Health Qual Life Outcomes 2018;16:89.

20. Maier W, Buller R, Philipp M, et al. The Hamilton Anxiety Scale: reliability, validity and sensitivity to change in anxiety and depressive disorders. J Affect Disord 1988;14:61-8. 


\section{The following six separate regression algorithms were used for the subretinal fluid absorption predictions:}

Decision Tree: Decision tree is a basic classification method with a tree structure. Classification problems can be regarded as sets of if-then rules. In every decision tree, all instances are covered by a path or rules. Generally, decision tree learning includes three steps: feature selection, decision tree generation, and decision tree pruning.

AdaBoost.R2: AdaBoost is a boosting algorithm based on the idea of fitting a sequence of weak learners by iterating over the same training set, and the final prediction results are obtained by calculating the weighted combination of the outputs of these weak learners. In the implementation of boosting algorithms, the weight of a sample with poor performance in the previous learner is increased, and the updated sample is then used to retrain the next weak learner. When combining all learners, the weight of each weak learner is decided based on its performance.

Gradient Boosting: Gradient Boosting is a generalization of boosting to arbitrary differentiable loss functions. In this method, the negative gradient of the loss function (the first derivative of the loss function) is used as a measure of the performance of a weak learner, and the weak learner is optimized by reducing the loss function in the direction of the gradient.

Extreme Gradient Boosting: Extreme Gradient Boosting (XGBoost) is an optimized distributed gradient boosting algorithm implemented based on the original Gradient Boosting framework. Instead of the first derivative, which is used in Gradient Boosting, the first-order and second-order Taylor expansions of the loss function are used in the optimization process in XGBoost. Consequently, its accuracy is higher, and fewer iterations are required to achieve satisfactory results. Unlike other boosting methods, XGBoost is able to use multithreading when choosing the best segmentation point. The parallel tree boosting operation substantially reduces the run time.

Random Forest: The Random Forest algorithm is a variant of the bagging (Bootstrap AGGregatING) algorithm that obtains its final results by averaging the predictions of many decision trees. The training set used to construct each decision tree is obtained using the bootstrap method (random sampling with replacement from the original data). Furthermore, when splitting one node during the construction of a tree, a subset of all features at that node is randomly selected, and then an optimal feature is selected from this subset for splitting. Because of the use of random sampling and random feature selection, the Random Forest algorithm is not easily susceptible to overfitting, although no pruning is performed on any single tree.

Extra-Trees: The Extremely Randomized Trees (Extra-Trees) algorithm is a variant of the Random Forest algorithm obtained by introducing random thresholds when splitting nodes. The Random Forest algorithm uses the bootstrap method to obtain the training set, whereas Extra-Trees uses all samples for training. Instead of choosing the most discriminative thresholds in feature subsets, as in the Random Forest algorithm, Extra-Trees randomly selects thresholds for the candidate features and then selects the best threshold for node splitting. 
Table S1 Clinical and Imaging Features Used to Predict Subretinal Fluid Absorption

\begin{tabular}{|c|c|c|c|c|c|c|c|}
\hline \multicolumn{4}{|c|}{ Clinical data } & \multicolumn{2}{|c|}{ Features from FFA and ICGA } & \multicolumn{2}{|r|}{ Features from OCTA } \\
\hline Feature & Description & Feature & Description & Feature & Description & Feature & Description \\
\hline Age & Age of the patient & Type-A Behavior & Type-A Behavior ${ }^{3}$ score & FFA leakage & Existence of active leakage at baseline & High reflection & Existence of high reflection at baseline \\
\hline Sex & Sex of the patient & Eye & Right or left eye & Single or multiple & No. of active leakage sites on FFA & Position 4 & Position of high reflection on OCTA \\
\hline Height & Height of the patient & Duration & Duration of CSC & Morphology & Morphology of the leakage on FFA & Low reflection & Existence of low reflection at baseline \\
\hline Weight & Weight of the patient & Therapy & hd-PDT, SML or CL & Area & Area of the leakage on FFA & Position 5 & Position of low reflection on OCTA \\
\hline Education & Education level of the patient & VA Baseline & VA before treatment & Position 1 & Position of the leakage on FFA & BVN Baseline & Existence of BVN at baseline \\
\hline Income & Income level of the patient & VA $1 \mathrm{M}$ & VA at $1 \mathrm{M}$ after treatment & ICGA leakage & Existence of active leakage at baseline & Position 6 & Position of BVN at baseline \\
\hline Heart Disease & History of heart disease & VA $3 \mathrm{M}$ & VA at $3 \mathrm{M}$ after treatment & High permeability & High permeability on ICGA & BVN $1 \mathrm{M}$ & Existence of BVN at $1 \mathrm{M}$ \\
\hline Gastropathy & History of gastropathy & VA $6 \mathrm{M}$ & VA at $6 \mathrm{M}$ after treatment & Position 2 & Position of the high permeability on ICGA & Position 7 & Position of BVN at $1 \mathrm{M}$ \\
\hline Autoimmune Disease & History of autoimmune disease & & & Low permeability & Low permeability on ICGA & BVN $3 \mathrm{M}$ & Existence of BVN at $3 \mathrm{M}$ \\
\hline Steroid Usage & History of steroid use & & & Position 3 & Position of the low permeability on ICGA & Position 8 & Position of BVN at $3 \mathrm{M}$ \\
\hline Hamilton Anxiety Scale & Hamilton Anxiety Scale'score & & & & & BVN $6 \mathrm{M}$ & Existence of BVN at $6 \mathrm{M}$ \\
\hline Pittsburgh Sleep Quality Index & x Pittsburgh Sleep Quality Index ${ }^{2}$ score & & & & & Position 9 & Position of BVN at $6 \mathrm{M}$ \\
\hline \multicolumn{2}{|c|}{ Features from OCT (Baseline) } & \multicolumn{2}{|r|}{ Features from OCT $(1 \mathrm{M})$} & \multicolumn{2}{|r|}{ Features from OCT $(3 \mathrm{M})$} & \multicolumn{2}{|r|}{ Features from OCT $(6 \mathrm{M})$} \\
\hline Feature & Description & Feature & Description & Feature & Description & Feature & Description \\
\hline SFA horizontal & Subretinal fluid absorption on horizontal B-scan & SFA horizontal & Subretinal fluid absorption on horizontal B-scan & SFA horizontal & Subretinal fluid absorption on horizontal B-scan & SFA horizontal & Subretinal fluid absorption on horizontal B-scan \\
\hline SFA vertical & Subretinal fluid absorption on vertical B-scan & SFA vertical & Subretinal fluid absorption on vertical B-scan & SFA vertical & Subretinal fluid absorption on vertical B-scan & SFA vertical & Subretinal fluid absorption on vertical B-scan \\
\hline SFA & Subretinal fluid absorption at baseline & SFA & Subretinal fluid absorption at $1 \mathrm{M}$ & SFA & Subretinal fluid absorption at $3 \mathrm{M}$ & SFA & Subretinal fluid absorption at $6 \mathrm{M}$ \\
\hline CMT horizontal & CMT on horizontal B-scan & CMT horizontal & CMT on horizontal B-scan & CMT horizontal & CMT on horizontal B-scan & CMT horizontal & CMT on horizontal B-scan \\
\hline CMT vertical & CMT on vertical B-scan & CMT vertical & CMT on vertical B-scan & CMT vertical & CMT on vertical B-scan & CMT vertical & CMT on vertical B-scan \\
\hline CMT & Average CMT at baseline & CMT & Average CMT at $1 \mathrm{M}$ & CMT & Average $\mathrm{CMT}$ at $3 \mathrm{M}$ & CMT & Average CMT at $6 \mathrm{M}$ \\
\hline RNEL horizontal & Thickness of RNEL on horizontal B-scan & RNEL horizontal & Thickness of RNEL on horizontal B-scan & RNEL horizontal & Thickness of RNEL on horizontal B-scan & RNEL horizontal & Thickness of RNEL on horizontal B-scan \\
\hline RNEL vertical & Thickness of RNEL on vertical B-scan & RNEL vertical & Thickness of RNEL on vertical B-scan & RNEL vertical & Thickness of RNEL on vertical B-scan & RNEL vertical & Thickness of RNEL on vertical B-scan \\
\hline RNEL & Average thickness of RNEL at baseline & RNEL & Average thickness of RNEL at $1 \mathrm{M}$ & RNEL & Average thickness of RNEL at $3 \mathrm{M}$ & RNEL & Average thickness of RNEL at $6 \mathrm{M}$ \\
\hline SRF horizontal & Height of SRF on horizontal B-scan & SRF horizontal & Height of SRF on horizontal B-scan & SRF horizontal & Height of SRF on horizontal B-scan & SRF horizontal & Height of SRF on horizontal B-scan \\
\hline SRF vertical & Height of SRF on vertical B-scan & SRF vertical & Height of SRF on vertical B-scan & SRF vertical & Height of SRF on vertical B-scan & SRF vertical & Height of SRF on vertical B-scan \\
\hline SRF & Average height of SRF at baseline & SRF & Average height of SRF at $1 \mathrm{M}$ & SRF & Average height of SRF at $3 \mathrm{M}$ & SRF & Average height of SRF at $6 \mathrm{M}$ \\
\hline ChT horizontal & ChT on horizontal B-scan & ChT horizontal & ChT on horizontal B-scan & $n T$ horizontal & $\mathrm{ChT}$ on horizontal B-scan & izontal & ChT on horizontal B-scan \\
\hline ChT vertical & ChT on vertical B-scan & ChT vertical & ChT on vertical B-scan & $h$ T vertical & $\mathrm{ChT}$ on verti $\mathrm{CH}$ & ChT vertical & $\mathrm{ChT}$ on vertical B-scan \\
\hline & Average $\mathrm{ChT}$ at baseline & ChT & Average $\mathrm{Ch}$ & ${ }^{2}$ & Average $\mathrm{C}$ & $\mathrm{ChT}$ & Average $\mathrm{ChT}$ at $6 \mathrm{M}$ \\
\hline EZ horizontal & Integrity of EZ on horizontal B-scan & $\mathrm{ChT}$ (1 M-B) horizontal & $\mathrm{ChT}$ variation (1M-baseline) on horizontal B-scan & IT (3 M-1 M) horizontal & ChT variation $(3 \mathrm{M}-1 \mathrm{M})$ on horizontal $\mathrm{B}-\mathrm{sccan}$ & ChT (6 M-3 M) horizontal & ChT variation $(6 \mathrm{M}-3 \mathrm{M})$ on horizontal $\mathrm{B}$-scan \\
\hline EZ vertical & Integrity of EZ on vertical B-scan & ChT (1 M-B) vertical & ChT variation (1 M-baseline) on vertical B-scan & $\mathrm{ChT}(3 \mathrm{M}-1 \mathrm{M})$ vertical & ChT variation $(3 \mathrm{M}-1 \mathrm{M})$ on vertical $\mathrm{B}$-scan & $\mathrm{ChT}(6 \mathrm{M}-3 \mathrm{M})$ vertical & ChT variation $(6 \mathrm{M}-3 \mathrm{M})$ on vertical B-scan \\
\hline & Average integrity of $E Z$ at baseline & $\mathrm{ChT}(1 \mathrm{M}-\mathrm{B})$ & Average $\mathrm{ChT}$ variation (1 M-baseline) at $1 \mathrm{M}$ & $\mathrm{ChT}(3 \mathrm{M}-1 \mathrm{M})$ & Average $\mathrm{ChT}$ variation $(3 \mathrm{M}-1 \mathrm{M})$ at $3 \mathrm{M}$ & $\mathrm{ChT}(6 \mathrm{M}-3 \mathrm{M})$ & Average ChT variation $6 \mathrm{M}-$ \\
\hline PED horizontal & Existence of PED on horizontal B-scan & EZ horizontal & Integrity of EZ on horizontal B-scan & EZ horizontal & Integrity of EZ on horizontal B-scan & EZ horizontal & Integrity of EZ on \\
\hline PED vertical & Existence of PED on vertical B-scan & EZ vertical & Integrity of EZ on vertical B-scan & EZ vertical & Integrity of EZ on vertical B-scan & EZ vertical & Integrity of EZ on vertical B-scan \\
\hline & Existence of PED at baseline & & Average integrity of $\mathrm{EZ}$ at $1 \mathrm{M}$ & & Average integrity of $\mathrm{EZ}$ at $3 \mathrm{M}$ & & Average integrity of $E Z$ at $6 \mathrm{M}$ \\
\hline DLS horizontal & Existence of DLS on horizontal B-scan & PED horizontal & Existence of PED on horizontal B-scan & PED horizontal & Existence of PED on horizontal B-scan & PED horizontal & Existence of PED on horizontal B-scan \\
\hline DLS vertical & Existence of DLS on vertical B-scan & PED vertical & Existence of PED on vertical B-scan & PED vertical & Existence of PED on vertical B-scan & PED vertical & Existence of PED on vertical B-scan \\
\hline & Existence of DLS at baseline & PED & Existence of PED at $1 \mathrm{M}$ & PED & Existence of PED at $3 \mathrm{M}$ & PED & Existence of PED at $6 \mathrm{M}$ \\
\hline Bruch's membrane horizontal & Bruch's membrane on horizontal B-scan & DLS horizontal & Existence of DLS on horizontal B-scan & DLS horizontal & Existence of DLS on horizontal B-scan & DLS horizontal & Existence of DLS on horizontal B-scan \\
\hline \multirow{8}{*}{ Bruch's membrane } & Bruch's membrane on vertical B-scan & DLS vertical & Existence of DLS on vertical B-scan & DLS vertical & Existence of DLS on vertical B-scan & DLS vertical & Existence of DLS on vertical B-scan \\
\hline & Bruch's membrane at baseline & & Existence of DLS at $1 \mathrm{M}$ & DLS & Existence of DLS at $3 \mathrm{M}$ & & Existence of DLS at $6 \mathrm{M}$ \\
\hline & & Bruch's membrane horizontal & Bruch's membrane on horizontal B-scan & Bruch's membrane horizontal & Bruch's membrane on horizontal B-scan & Bruch's membrane horizontal & ruch's membrane on horizontal B-scan \\
\hline & & Bruch's membrane vertical & Bruch's membrane on vertical B-scan & Bruch's membrane vertical & Bruch's membrane on vertical B-scan & Bruch's membrane vertical & Bruch's membrane on vertical B-scan \\
\hline & & Bruch's membrane & Bruch's membrane at $1 \mathrm{M}$ & Bruch's membrane & Bruch's membrane at $3 \mathrm{M}$ & Bruch's membrane & Bruch's membrane at $6 \mathrm{M}$ \\
\hline & & & & Recurrence horizontal & Recurrence on horizontal B-scan & Recurrence horizontal & Recurrence on horizontal B-scan \\
\hline & & & & Recurrence vertical & Recurrence on vertical B-scan & Recurrence vertical & Recurrence on vertical B-scan \\
\hline & & & & Recurrence & Recurrence at $3 \mathrm{M}$ & Recurrence & Recurrence at $6 \mathrm{M}$ \\
\hline
\end{tabular}


This table shows all 20 clinical features and 145 imaging features used to predict SFA. Twenty features (e.g., the duration of CSC) were retrieved from the electronic medical records, 5 features (e.g., position and area of the leakage point) were calculated from FFA, 5 features (e.g., hyperperfusion and hypoperfusion) were calculated from ICGA, 12 features (e.g., the existence of abnormal reflection and branching vascular network [BVN]) were calculated from OCTA and 123 features (e.g., RNEL, CMT, and EZ) were calculated from OCT. SFA, subretinal fluid absorption; OCT, optical coherence tomography; OCTA, optical coherence tomography angiography; CSC, central serous chorioretinopathy; CL, conventional laser; SML, subthreshold micropulse laser; hd-PDT, half-dose photodynamic therapy; FFA, fundus fluorescein angiography; single or multiple, a label of 1 indicates the existence of a single leakage point and 2 indicates multiple leakage sites; Morphology, a label of 1 indicates smokestack leakage on FFA, 2 indicates focal diffuse leakage, and 3 indicates multiple diffuse leakage sites; area, a label of 1 indicates that the leakage area on FFA was less than the area of the optic disc and 2 indicates a larger area; position (position 1 to position 9), a label of 1 indicates that the damage was located less than 1500 microns away from the fovea and 2 indicates a distance greater than 1500 microns; ICGA, indocyanine green angiography; high permeability, a label of 1 indicates the existence of high permeability and 2 indicates normal permeability; low permeability, a label of 1 indicates the existence of low permeability and 2 indicates normal permeability; high reflection, a label of 1 indicates the existence of high reflection on OCTA and 2 indicates normal reflection; low reflection, a label of 1 indicates the existence of low reflection on OCTA and 2 indicates normal reflection; and BVN, a label of 1 indicates the existence of BVN and 2 indicates a normal structure. All the OCTA features are derived from images of the superficial choroidal layer, defined as 10 microns above the Bruch's membrane to 30 microns below the Bruch's membrane in the $3^{*} 3$ scanning pattern of Optovue (version 2017.1.0.155) software. SRF, subretinal fluid; CMT, central macular thickness; RNEL, retinal neuroepithelial layer; ChT, choroidal thickness, all measurements are expressed in microns; SFA, a label of 1 indicates an increase in the level of unabsorbed SRF, 2 indicates partially absorbed SRF, and 3 indicates completely absorbed SRF; EZ, ellipsoid zone, a label of 1 indicates the complete absence of the original neurosensory retinal detachment area, 2 indicates the intermittent existence of the original neurosensory retinal detachment area with less than half of the total length, 3 indicates the existence of most of the original neurosensory retinal detachment area and 4 indicates the complete existence of original neurosensory retinal detachment area; PED, retinal pigment epithelial detachment, a label of 1 indicates the existence of PED and 2 indicates a normal structure; DLS, double-layer sign, a label of 1 indicates the existence of DLS and 2 indicates a normal structure; Bruch's membrane, a label of 1 indicates the disruption of Bruch's membrane and 2 indicates a normal membrane; and recurrence, a label of 1 indicates the reappearance of SRF and 2 indicates a normal structure on OCT (in the analysis of quantitative data, we used the mean values of horizontal and vertical B-scans on OCT; in the analysis of qualitative data, we used the worse values of the horizontal and vertical B-scans on OCT).

1. Maier W, Buller R, Philipp M, et al. The Hamilton Anxiety Scale: reliability, validity and sensitivity to change in anxiety and depressive disorders. J Affect Disord. 1988;14(1):61-68.

2. Manzar MD, BaHammam AS, Hameed UA, et al. Dimensionality of the Pittsburgh Sleep Quality Index: a systematic review. Health Qual Life Outcomes. 2018;16(1):89.

3. Yannuzzi LA. Type-A behavior and central serous chorioretinopathy. Retina. 1987;7(2):111-131 
Table S2 Clinical and Imaging Features Used to Predict Subretinal Fluid Absorption in the Simplified Model

\begin{tabular}{|c|c|c|c|c|c|c|c|}
\hline \multicolumn{8}{|c|}{ Clinical data } \\
\hline Feature & Description & Feature & Description & Feature & Description & Feature & Description \\
\hline & Age of the patient & Hamilton Anxiety Scale & Hamilton Anxiety Scale ${ }^{1}$ score & Therapy & CL, SML, or hd-PDT & VA $3 M$ & VA at $3 \mathrm{M}$ after treatment \\
\hline Education & Education level of the patient & Pittsburgh Sleep Quality Index & Pittsburgh Sleep Quality Index ${ }^{2}$ score & VA Baseline & VA before treatment & VA $6 \mathrm{M}$ & VA at $6 \mathrm{M}$ after treatment \\
\hline \multirow[t]{2}{*}{ 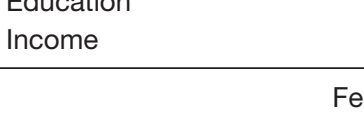 } & Income level of the patient & Duration & Duration of CSC & VA $1 \mathrm{M}$ & $\mathrm{VA}$ at $1 \mathrm{M}$ after treatment & & \\
\hline & $\begin{array}{l}\text { Income level of the patient } \\
\text { eatures from OCT (Baseline) }\end{array}$ & \multicolumn{2}{|r|}{ Features from OCT (1 M) } & \multicolumn{2}{|r|}{ Features from OCT $(3 \mathrm{M})$} & \multicolumn{2}{|r|}{ Features from $\mathrm{OCT}(6 \mathrm{M})$} \\
\hline Feature & Description & Feature & Description & Feature & Description & Feature & Description \\
\hline SFA horizontal & Subretinal fluid absorption on horizontal B-scan & SFA horizontal & Subretinal fluid absorption on horizontal B-scan & SFA horizontal & Subretinal fluid absorption on horizontal B-scan & SFA horizontal & Subretinal fluid absorption on horizontal B-scan \\
\hline SFA vertical & Subretinal fluid absorption on vertical B-scan & SFA vertical & Subretinal fluid absorption on vertical $\mathrm{B}$-scan & SFA vertical & Subretinal fluid absorption on vertical B-scan & SFA vertical & Subretinal fluid absorption on vertical B-scan \\
\hline $\begin{array}{l}\text { SFA } \\
\text { SFItid }\end{array}$ & Subretinal fluid absorption at baseline & SFA & Subretinal fluid absorption at $1 \mathrm{M}$ & SFA & Subretinal fluid absorption at $3 \mathrm{M}$ & SFA & Subretinal fluid absorption at $6 \mathrm{M}$ \\
\hline CMT horizontal & CMT on horizontal B-scan & CMT horizontal & $\begin{array}{l}\text { CMT on horizontal B-scan } \\
\text { Cor }\end{array}$ & CMT horizontal & $\begin{array}{l}\text { CMT on horizontal B-scan } \\
\text { Cor }\end{array}$ & CMT horizontal & CMT on horizontal B-scan \\
\hline $\begin{array}{l}\text { CMT vertical } \\
\text { Cil }\end{array}$ & $\begin{array}{l}\text { CMT on vertical B-scan } \\
\text { C-scan }\end{array}$ & $\begin{array}{l}\text { CMT vertical } \\
\text { CMT }\end{array}$ & CMT on vertical $\mathrm{B}$-scan & $\begin{array}{l}\text { CMT vertical } \\
\text { CMal }\end{array}$ & $\begin{array}{l}\text { CMT on vertical } B \text {-scan } \\
\text { Conan }\end{array}$ & $\begin{array}{l}\text { CMT vertical } \\
\text { Cal }\end{array}$ & $\begin{array}{l}\text { CMT on vertical } B \text {-scan } \\
\text { CScan }\end{array}$ \\
\hline $\begin{array}{l}\text { CMT vertical } \\
\text { CMT }\end{array}$ & 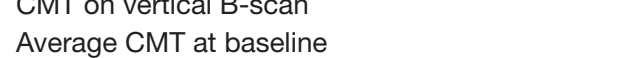 & $\begin{array}{l}\text { CMI vertical } \\
\text { CMT }\end{array}$ & $\begin{array}{l}\text { Average } \mathrm{CMT} \text { at } 1 \mathrm{M} \\
\text { Aven }\end{array}$ & $\begin{array}{l}\text { CM vertical } \\
\text { CMT }\end{array}$ & $\begin{array}{l}\text { Average } \mathrm{CMT} \text { at } 3 \mathrm{M} \\
\text { Avan }\end{array}$ & $\begin{array}{l}\text { CMM vertical } \\
\text { CMT }\end{array}$ & $\begin{array}{l}\text { CMI on vertical } \mathrm{B}-\mathrm{sc} \text { an } \\
\text { Average } \mathrm{CMT} \text { at } 6 \mathrm{M}\end{array}$ \\
\hline RNEL horizontal & $\begin{array}{l}\text { Average CMII at baseline } \\
\text { Thickness of RNEL on horizontal B-scan }\end{array}$ & RNEL horizontal & $\begin{array}{l}\text { Therage CiVI Ia T M } \\
\text { Thickness of RNEL on horizontal B-scan }\end{array}$ & $\begin{array}{l}\text { CMII horizontal } \\
\text { RNEL he }\end{array}$ & $\begin{array}{l}\text { TVerage CMII I } 3 \text { Th } \\
\text { Thickness of RNEL on horizontal B-scan }\end{array}$ & RNEL horizontal & $\begin{array}{l}\text { Average CMI at } 6 \text { M } \\
\text { Thickness of RNEL on horizor }\end{array}$ \\
\hline $\begin{array}{l}\text { RINEL horizontal } \\
\text { RNEL vertical }\end{array}$ & $\begin{array}{l}\text { Thickness of RNEL on horzizontal B-scann } \\
\text { Thickness of RNEL on vertical B-scan }\end{array}$ & $\begin{array}{l}\text { RNELL verizontal } \\
\text { RNEal }\end{array}$ & $\begin{array}{l}\text { IIIckness of RNEL on horzontal B-scan } \\
\text { Thickness of RNEL on vertical B-scan }\end{array}$ & $\begin{array}{l}\text { RNEL horizontal } \\
\text { RNEL vertical }\end{array}$ & $\begin{array}{l}\text { Thickness of RNEL on horizontal B-scan } \\
\text { Thickness of RNEL on vertical B-scan }\end{array}$ & $\begin{array}{l}\text { RNEL horizontal } \\
\text { RNEL vertical }\end{array}$ & $\begin{array}{l}\text { Inickness of RNNEL on horzzontal B-scan } \\
\text { Thickness of RNEL on vertical B-scan }\end{array}$ \\
\hline RNEL & Average thickness of RNEL at baseline & RNEL & Average thickness of RNEL at $1 \mathrm{M}$ & RNEL & Average thickness of RNEL at $3 \mathrm{M}$ & RNEL & Average thickness of RNEL at $6 \mathrm{M}$ \\
\hline SRF horizontal & Height of SRF on horizontal B-scan & SRF horizontal & Height of SRF on horizontal B-scan & SRF horizonta & Height of SRF on horizontal B-scan & SRF horizonta & Height of SRF on horizontal B-scan \\
\hline $\begin{array}{l}\text { SRF vertical } \\
\text { Stats }\end{array}$ & Height of SRF on vertical B-scan & SRF vertical & Height of SRF on vertical B-scan & SRF vertical & Height of SRF on vertical B-scan & SRF vertical & Height of SRF on vertical B-scan \\
\hline & Average height of SRF at baseline & $\mathrm{BF}$ & Average height of SRF at $1 \mathrm{M}$ & SRF & Average height of SRF at $3 \mathrm{M}$ & SRF & Average height of $S R F$ at $6 \mathrm{M}$ \\
\hline ChT horizontal & $\begin{array}{l}\mathrm{ChT} \text { on horizontal B-scan } \\
\mathrm{C} \text { - }\end{array}$ & ChT horizontal & $\begin{array}{l}\text { ChT on horizontal B-scan } \\
\text { Cht }\end{array}$ & ChT horizontal & ChT on horizontal B-scan & ChT horizontal & ChT on horizontal B-scan \\
\hline ChT vertical & $\mathrm{ChT}$ on vertical B-scan & $\mathrm{ChT}$ vertical & $\mathrm{ChT}$ on vertical B-scan & $\mathrm{ChT}$ vertical & ChT on vertical B-scan & ChT vertical & ChT on vertical B-scan \\
\hline & Average $\mathrm{ChT}$ at baseline & ChT & Average $\mathrm{ChT}$ at $1 \mathrm{M}$ & $\mathrm{ChT}$ & Average $\mathrm{ChT}$ at $3 \mathrm{M}$ & $\mathrm{ChT}$ & Average $\mathrm{ChT}$ at $6 \mathrm{M}$ \\
\hline EZ horizontal & Integrity of EZ on horizontal B-scan & ChT (1 M-B) horizontal & ChT variation (1M-baseline) on horizontal B-scan & $\mathrm{ChT}(3 \mathrm{M}-1 \mathrm{M})$ horizontal & $\mathrm{ChT}$ variation (3 M-1 M) on horizontal $\mathrm{B}-\mathrm{scan}$ & $\mathrm{ChT}(6 \mathrm{M}-3 \mathrm{M})$ horizontal & ChT variation (6 M-3 $\mathrm{M}$ ) on horizontal B-scan \\
\hline EZ vertical & Integrity of EZ on vertical B-scan & $\operatorname{ChT}(1 \mathrm{M}-\mathrm{B})$ vertical & ChT variation (1 M-baseline) on vertical B-scan & ChT $(3 \mathrm{M}-1 \mathrm{M})$ vertical & $C h T$ variation (3 M-1 M) on vertical B-scan & ChT (6 M-3 M) vertical & ChT variation (6 M-3 M) on vertical $\mathrm{B}-\mathrm{sccan}$ \\
\hline & Average integrity of $E Z$ at baseline & $\operatorname{ChT}(1 \mathrm{M}-\mathrm{B})$ & Average $\mathrm{ChT}$ variation (1 M-baseline) at $1 \mathrm{M}$ & $\mathrm{ChT}(3 \mathrm{M}-1 \mathrm{M})$ & Average $C h T$ variation $(3 \mathrm{M}-1 \mathrm{M})$ at $3 \mathrm{M}$ & ChT $(6 \mathrm{M}-3 \mathrm{M})$ & Average $\mathrm{ChT}$ variation $(6 \mathrm{M}-3 \mathrm{M})$ at $6 \mathrm{M}$ \\
\hline PED horizontal & Existence of PED on horizontal B-scan & EZ horizontal & Integrity of EZ on horizontal B-scan & EZ horizontal & Integrity of EZ on horizontal B-scan & EZ horizontal & Integrity of EZ on horizontal B-scan \\
\hline PED vertical & Existence of PED on vertical B-scan & EZ vertical & Integrity of EZ on vertical B-scan & EZ vertical & Integrity of $E Z$ on vertical B-scan & EZ vertical & Integrity of EZ on vertical B-scan \\
\hline PED & Existence of PED at baseline & & Average integrity of $E Z$ at $1 \mathrm{M}$ & & Average integrity of $E Z$ at $3 \mathrm{M}$ & & Average integrity of $E Z$ at $6 \mathrm{M}$ \\
\hline DLS horizontal & Existence of DLS on horizontal B-scan & PED horizontal & Existence of PED on horizontal B-scan & PED horizontal & Existence of PED on horizontal B-scan & PED horizontal & Existence of PED on horizontal B-scan \\
\hline DLS vertical & Existence of DLS on vertical B-scan & PED vertical & Existence of PED on vertical B-scan & PED vertical & Existence of PED on vertical B-scan & PED vertical & Existence of PED on vertical B-scan \\
\hline & Existence of DLS at baseline & PED & Existence of PED at $1 \mathrm{M}$ & PED & Existence of PED at $3 \mathrm{M}$ & PED & Existence of PED at $6 \mathrm{M}$ \\
\hline Bruch's membrane horizontal & Bruch's membrane on horizontal B-scan & DLS horizontal & Existence of DLS on horizontal B-scan & DLS horizontal & Existence of DLS on horizontal B-scan & DLS horizontal & Existence of DLS on horizontal B-scan \\
\hline Bruch's membrane vertical & Bruch's membrane on vertical B-scan & DLS vertical & Existence of DLS on vertical B-scan & DLS vertical & Existence of DLS on vertical B-scan & DLS vertical & Existence of DLS on vertical B-scan \\
\hline \multirow[t]{7}{*}{ Bruch's membrane } & Bruch's membrane at baseline & & Existence of DLS at $1 \mathrm{M}$ & DLS & Existence of DLS at $3 \mathrm{M}$ & & Existence of DLS at $6 \mathrm{M}$ \\
\hline & & Bruch's membrane horizontal & Bruch's membrane on horizontal B-scan & Bruch's membrane horizontal & Bruch's membrane on horizontal B-scan & Bruch's membrane horizontal & Bruch's membrane on horizontal B-scan \\
\hline & & Bruch's membrane vertical & Bruch's membrane on vertical B-scan & Bruch's membrane vertical & Bruch's membrane on vertical B-scan & Bruch's membrane vertical & Bruch's membrane on vertical B-scan \\
\hline & & Bruch's membrane & Bruch's membrane at $1 \mathrm{M}$ & Bruch's membrane & Bruch's membrane at $3 \mathrm{M}$ & Bruch's membrane & Bruch's membrane at $6 \mathrm{M}$ \\
\hline & & & & Recurrence horizontal & Recurrence on horizontal B-scan & Recurrence horizontal & Recurrence on horizontal B-scan \\
\hline & & & & Recurrence vertical & Recurrence on vertical B-scan & Recurrence vertical & Recurrence on vertical B-scan \\
\hline & & & & Recurrence & Recurrence at $3 \mathrm{M}$ & Recurrence & Recurrence at $6 \mathrm{M}$ \\
\hline
\end{tabular}


This table shows all 11 clinical features and 123 imaging features used to predict SFA in the simplified model. Eleven features (e.g., duration of CSC) were retrieved from the electronic medical records, and 123 features (e.g., RNEL, CMT, and EZ) were calculated from OCT. SFA, subretinal fluid absorption; OCT, optical coherence tomography; CSC, central serous chorioretinopathy; CL, conventional laser; SML, subthreshold micropulse laser; hd-PDT, half-dose photodynamic therapy; SRF, subretinal fluid; CMT, central macular thickness; RNEL, retinal neuroepithelial layer; ChT, choroidal thickness, all measurements are expressed in microns; SFA, a label of 1 indicates an increase in the level of unabsorbed SRF, 2 indicates partially absorbed SRF, and 3 indicates completely absorbed SRF; EZ, ellipsoid zone, a label of 1 indicates the complete absence of the original neurosensory retinal detachment area, 2 indicates the intermittent existence of the original neurosensory retinal detachment area with less than half of the total length, 3 indicates the existence of most of the original neurosensory retinal detachment area and 4 indicates the complete existence of original neurosensory retinal detachment area; PED, retinal pigment epithelial detachment, a label of 1 indicates the existence of PED and 2 indicates a normal structure; DLS, double-layer sign, a label of 1 indicates the existence of DLS and 2 indicates a normal structure; Bruch's membrane, a label of 1 indicates the disruption of Bruch's membrane and 2 indicates a normal membrane; and recurrence, a label of 1 indicates the reappearance of SRF and 2 indicates a normal structure on OCT (in the analysis of quantitative data, we used the mean values of horizontal and vertical B-scans on OCT; in the analysis of qualitative data, we used the worse value of the horizontal and vertical B-scans on OCT).

1. Maier W, Buller R, Philipp M, et al. The Hamilton Anxiety Scale: reliability, validity and sensitivity to change in anxiety and depressive disorders. J Affect Disord. 1988;14(1):61-68.

2. Manzar MD, BaHammam AS, Hameed UA, et al. Dimensionality of the Pittsburgh Sleep Quality Index: a systematic review. Health Qual Life Outcomes. 2018;16(1):89. 


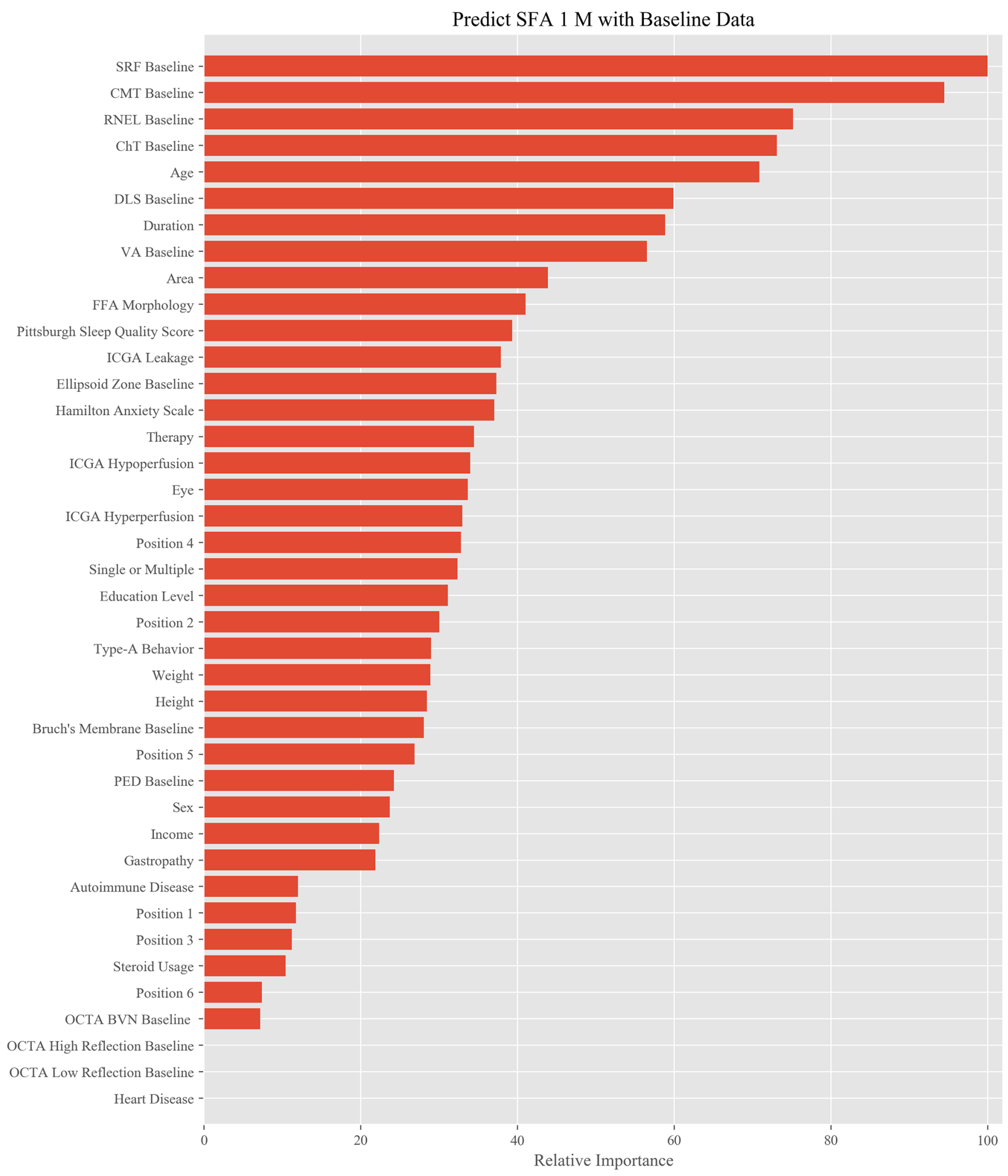

Figure S1 Relative Importance of Different Features to the $1 \mathrm{M}$ Prediction of the Full Model. This figure shows the relative importance of the baseline data in the SFA predictions. 


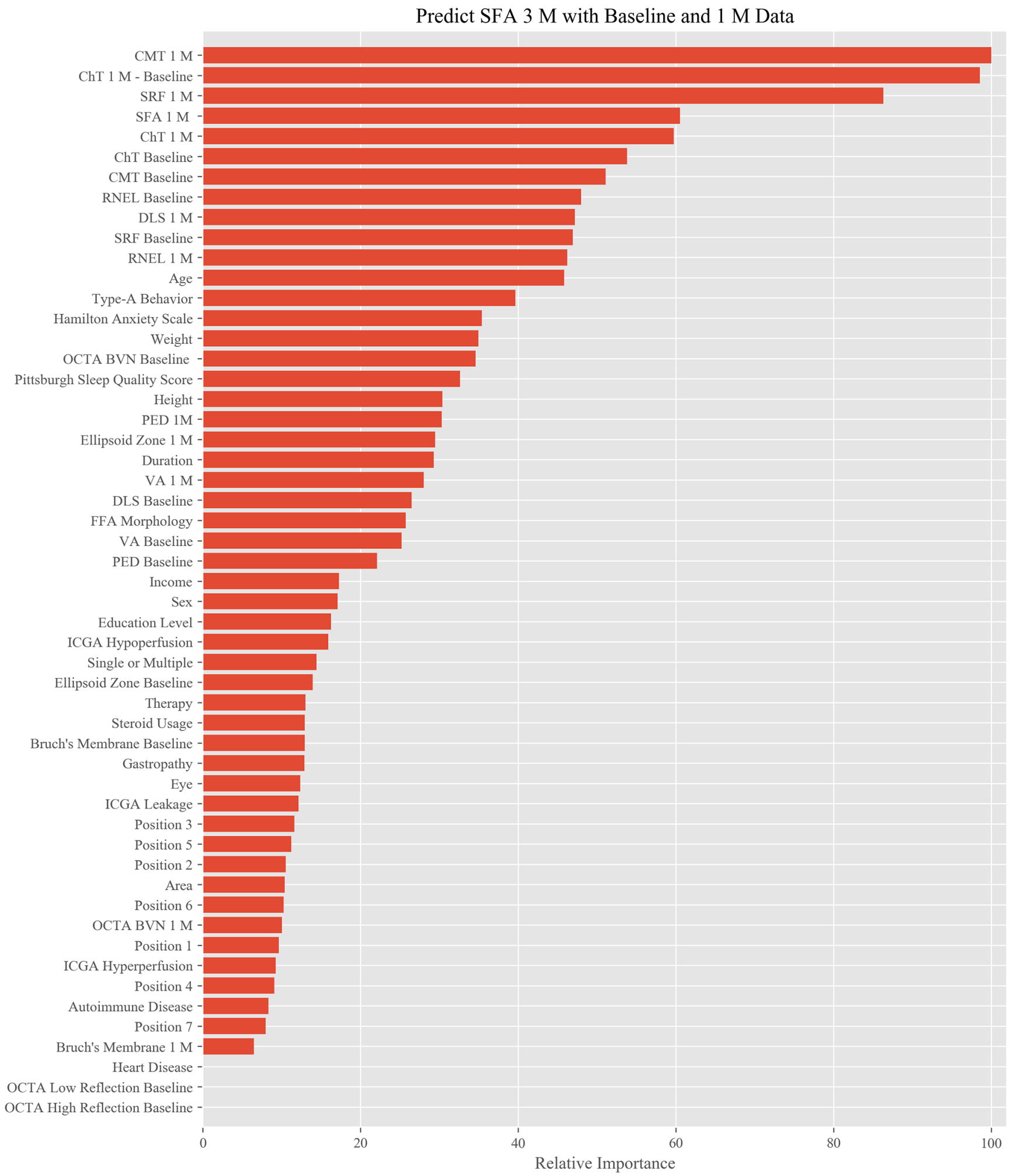

Figure S2 Relative Importance of Different Features to the 3 M Prediction of the Full Model. This figure shows the relative importance of the baseline and $1 \mathrm{M}$ data in the SFA predictions. 


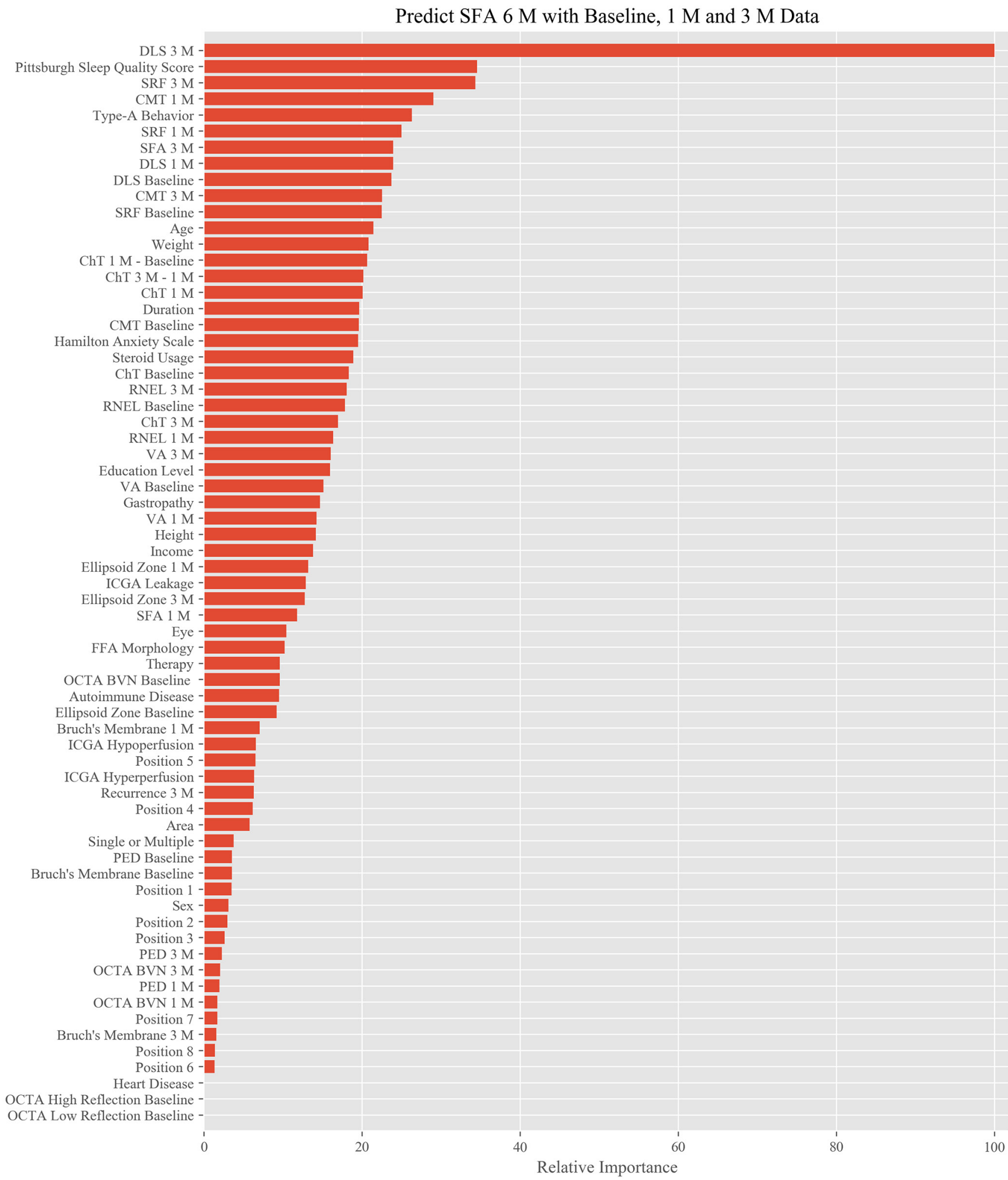

Figure S3 Relative Importance of Different Features to the $6 \mathrm{M}$ Prediction of the Full Model. This figure shows the relative importance of the baseline, $1 \mathrm{M}$ and $3 \mathrm{M}$ data in the SFA predictions. 


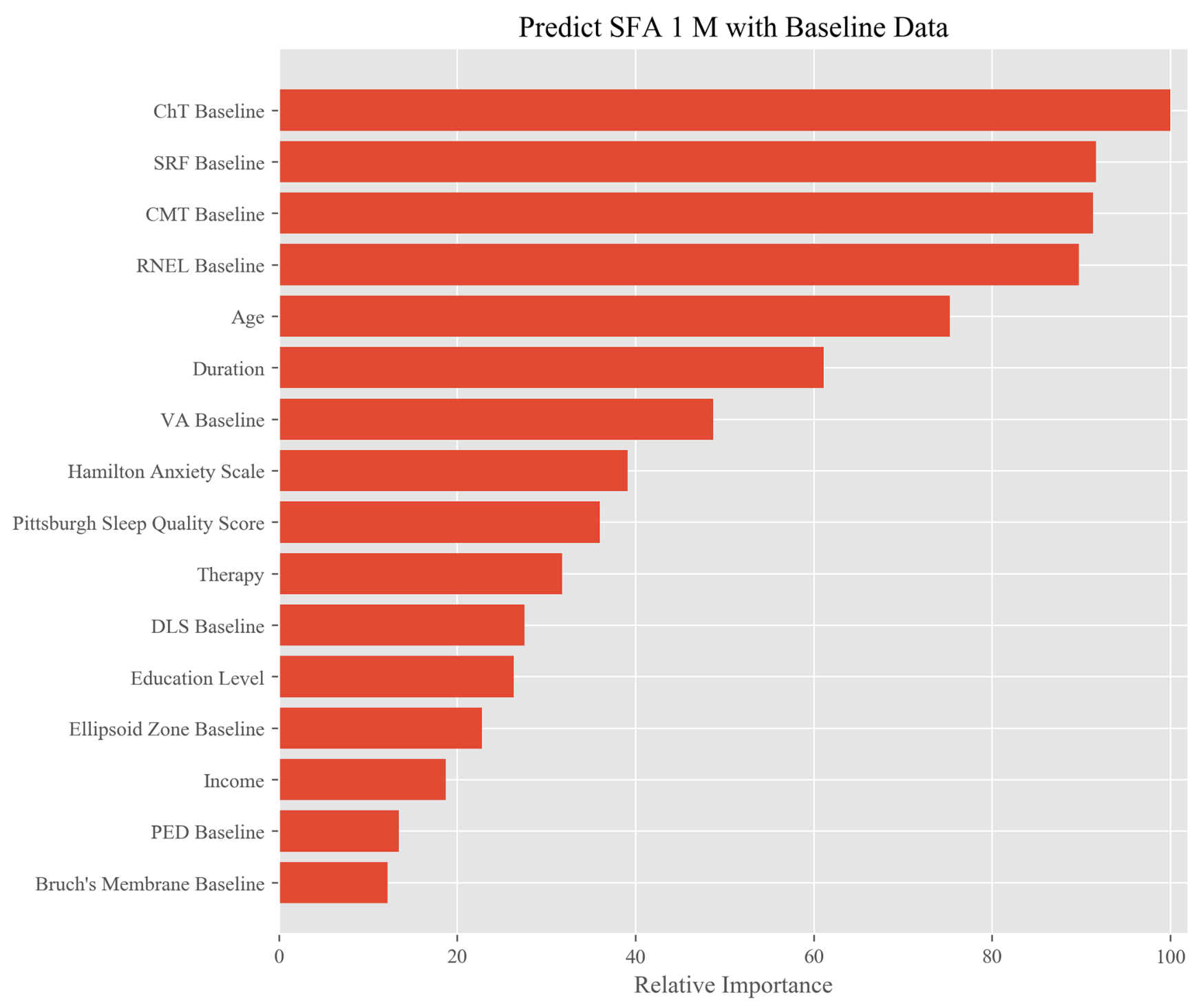

Figure S4 Relative Importance of Different Features to the $1 \mathrm{M}$ Prediction of the Simplified Model. This figure shows the relative importance of the baseline data in the SFA predictions. 




Figure S5 Relative Importance of Different Features to the 3 M Prediction of the Simplified Model. This figure shows the relative importance of the baseline and $1 \mathrm{M}$ data in the SFA predictions. 


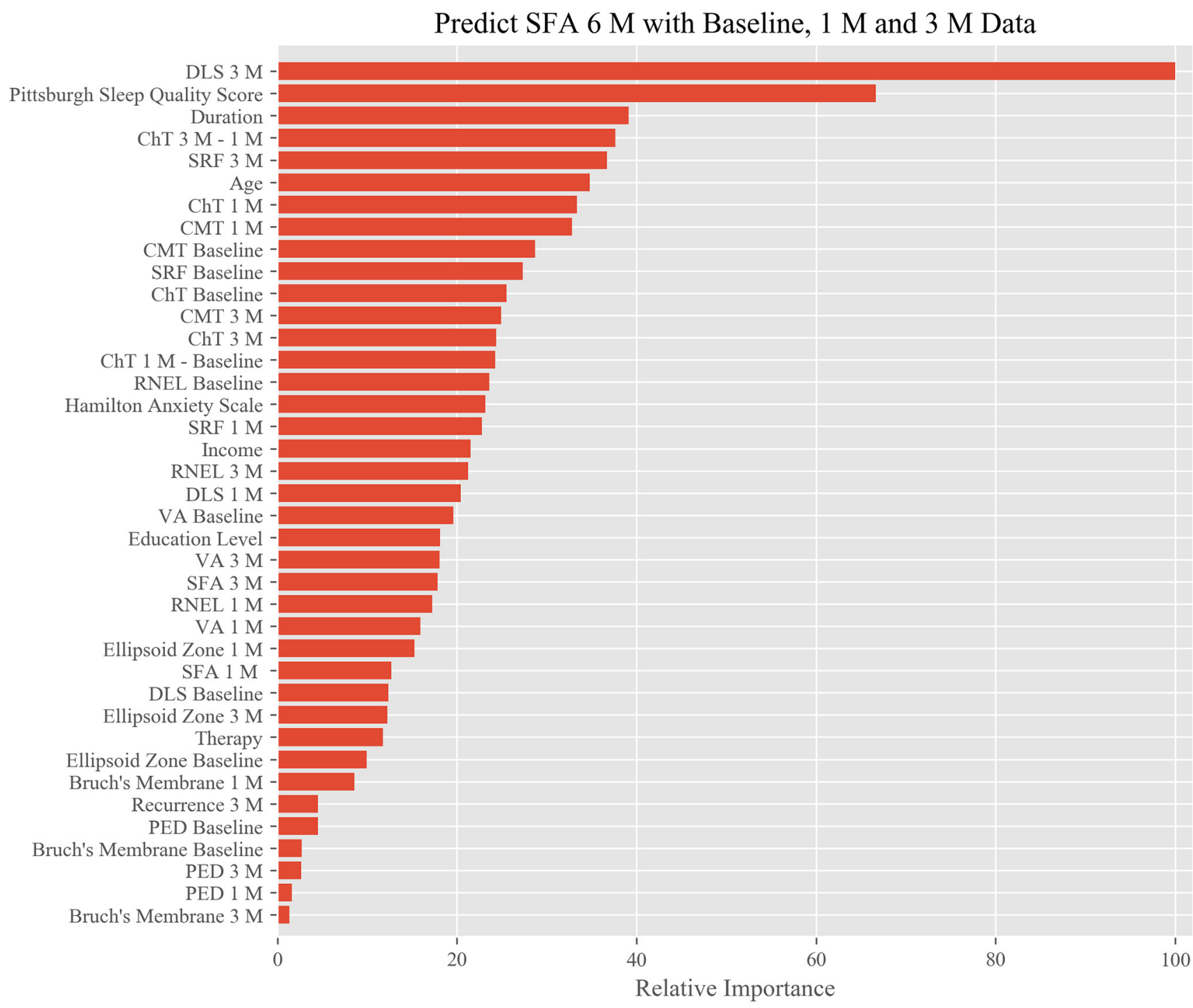

Figure S6 Relative Importance of Different Features to the $6 \mathrm{M}$ Prediction of the Simplified Model. This figure shows the relative importance of the baseline, $1 \mathrm{M}$ and $3 \mathrm{M}$ data in the SFA predictions. 